Volume 9, No.3, May - June 2020

International Journal of Advanced Trends in Computer Science and Engineering

Available Online at http://www.warse.org/IJATCSE/static/pdf/file/ijatcse143932020.pdf https://doi.org/10.30534/ijatcse/2020/143932020

\title{
Geometric Feature Based Facial Emotion Recognition
}

\author{
Khalid Ounachad $^{1}$, Mohamed Oualla ${ }^{2}$, Abdelalim Sadiq ${ }^{3}$ \\ ${ }^{1,3}$ Department of Informatics, Faculty of sciences, Ibn Tofail University, Kenitra, Morocco, \\ khalid.ounachad@uit.ac.ma, a.sadiq@uit.ac.ma \\ ${ }^{2}$ SEISE: Software Engineering \& Information Systems Engineering Team, Faculty of sciences \& technology, \\ Moulay Ismail University, Errachidia, Morocco, mohamedoualla76@gmail.com
}

\begin{abstract}
Facial Emotion Recognition (FER) is an important topic in the fields of computer vision and artificial intelligence owing to its significant academic and commercial potential. Nowadays, emotional factors are important as classic functional aspects of customer purchasing behavior. Purchasing choices and decisions making are the result of a careful analysis of the product advantages and disadvantages and of affective and emotional aspects. This paper presents a novel method for human emotion classification and recognition. We generate seven referential faces suitable for each kind of facial emotion based on perfect face ratios and some classical averages. The basic idea is to extract perfect face ratios for emotional face and for each referential face as features and calculate the distance between them by using fuzzy hamming distance. To extract perfect face ratios, we use the point landmarks in the face then sixteen features will be extract. An experimental evaluation demonstrates the satisfactory performance of our approach on WSEFEP dataset (the Warsaw Set of Emotional Facial Expression Pictures dataset). It can be applied with any existing facial emotion dataset. The proposed algorithm will be a competitor of the other proposed relative approaches. The recognition rate reaches more than $93 \%$.
\end{abstract}

Key words: Average Face Ratios; Facial Emotion Recognition; Fuzzy Hamming distance; Perfect Face Ratios.

\section{INTRODUCTION}

People's face is the most exposed part of body. Emotion is one of the most important attributes of face. Emotions are used in the process of non-verbal communication. They help us to understand the interactions of others. People, can immediately recognize an emotional state of a person. They are seven basics kinds of emotions: Anger, Disgust, Fear, Happiness, Neutral, Sadness and Surprise [1]. The accurate emotion recognition enables you to perform emotion based, sophisticated tasks, such as advertising customers satisfaction analysis.
Face Emotion Recognition systems (FER) aim to recognize a face emotion in a dataset of photos images [2][3][4][5]. The task of emotion recognition is particularly difficult: There doesn't exist a large dataset of training images and classifying emotion can be also difficult. In a face emotion recognition system, the feature extraction is the core block. Matching is used to recognize the right kind of emotion using the precomputed features. The preprocessing step can boost the final performance of the system considerably. Feature extraction aims to transform the input face emotion image into a set of features. Matching is a general concept to describe the task of finding correspondences between two elements that has to be carried out for recognition or classification. It can attempt a simple comparison between the features extracted or more complex comparison systems by using some distances.

The objective of this paper is to propose our method based on fuzzy hamming distance with average face ratios to recognizing a face emotion. This paper presents a novel method for human emotion classification and recognition. We generate seven referential faces suitable for each kind of facial emotion based on perfect face ratios and five classical averages: arithmetic mean, geometric mean, harmonic mean, contraharmonic mean and quadratic mean. The basic idea is to extract perfect face ratios for emotional face and for each referential face as features and calculate the distance between them by using fuzzy hamming distance. To extract perfect face ratios, we use the point landmarks in the face then sixteen features will be extract. To compute distances between the input facial emotion image and each image in a set of our referential faces, the hamming distance based on logical exclusive-or (XOR) function is used.

The Hamming distance evaluates the number of bits that differ from two binary vectors. The Fuzzy Hamming distance [6] has been published to solve Hamming distance limitations on real numbers. This distance is used in this work because real values features are computed, not binary numbers. It ensures great performances in terms of speed and accuracy. This article is organized as follows. Section 2 related works and background information about four classical averages, 
perfect face ratios and fuzzy hamming distance, their formalisms and their definitions, section 3 , the proposed architecture of our approach is explained in depth. Experimental results are given in section 4 . In section 5 , a conclusion is presented.

\section{RELATED WORKS}

Over the last decades, there has been a wealth of research in hamming distance, as well as in its application in computer vision specially in Face Sketch recognition [7], in Banknote Validator [8] and in Content-Based Image Retrieval System (CBIRS) [9]:

In [7] we presented a new facial sketch recognition method based on fuzzy hamming distance with geometric relationships (face ratios). We proposed to simplify the procedure based on fuzzy hamming distance to use only vectors with simple reals values of characteristics. An interesting contribution of [7] is that can accurately recognize the photo of the sketch's face. The proposed algorithm will be a competitor of the other proposed relative approaches. The recognition rate reaches $100 \%$ especially in the CUHK dataset.

The Fuzzy Hamming Distance based approach, proposed in Banknote Validator [8], combines the versatility of an automatic system with basic banknote specific information. Subsequently, the system can be updated to use in-depth security features provided by an expert. fuzzy Hamming distance is used to measure the similarity between banknotes

The study suggested by M. Ionescu et al. [9][10] presented initial results on a new approach to measure similarity between images using the notion of Fuzzy Hamming Distance (FHD) and its use to CBIR. The main advantage of the FHD is that the extent to which two different images are considered indeed different can be tuned to become more context dependent and to capture (implicit) semantic image information. The study shows good results using complete linkage agglomerative clustering. Fuzzy Hamming Distance proved to be efficient in a Content Based Image Retrieval system, that output the closest images in the database given a query image. In [10], the obtained results for image retrieval based on using hamming distance showed effectiveness of their approach.

In [11], character recognition is carried out using template matching scheme using correlation. In their approach [11], in addition to correlation, the hamming distance was applied in the scenarios where the correlation fails, the text image is segmented into lines and then characters by basic pre-processing techniques. After that, the character image is classified and converted into text by template matching scheme using either correlation or hamming distance.

\subsection{Contribution of this Work}

This article proposes a new facial emotion recognition method based on fuzzy Hamming distance with face ratios and their geometric relationships. Our work is inspired in part by the recent and successful method that has shown that relatively simple benchmark features could be used to perform well in a fuzzy Hamming distance-based face emotion recognition framework. In this paper, we simplify the procedure based on fuzzy hamming distance to use only vectors with simple reals values of characteristics. A key technical contribution of our paper is a method for recognizing a facial emotion based on these simple features that can accurately recognize the right kind of the emotion's face. This method achieves our goal by producing a recognition rate reaches more than $93 \%$.

\section{BACKGROUND INFORMATIONS}

Symbolically, we have a data set containing the values $x_{1}, x_{2}, \ldots, x_{n}$.

The arithmetic mean (Average) is equal to the sum of all numerical values of a set divided by the number of items in that set, then the average $\mathrm{A}$ is defined by the formula:

$$
A=\frac{1}{n} \sum_{i=1}^{n} x_{i}=\frac{s}{N}
$$

$N=$ The number of items being averaged.

$5=$ The sum of the numbers being averaged.

The geometric mean is the $n$th root of the product of $n$ numbers, for a set of numbers, then the average $G$ is defined by the formula:

$$
G=\left(\prod_{i=1}^{n} x_{i}\right)^{\frac{1}{n}}=\sqrt[n]{x_{1} x_{2} \ldots x_{n}}
$$

The harmonic mean is the reciprocal of the arithmetic mean of the reciprocals of the given numbers, then the average $\mathrm{H}$ is defined by the formula:

$$
H=\frac{n}{\sum_{i=1}=\frac{T}{x_{i}}}
$$

The contraharmonic mean is the arithmetic mean of the squares of the values divided by the arithmetic mean of tge values, then the average $\mathrm{C}$ is defined by the formula:

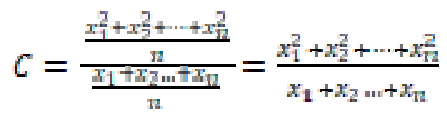

The quadratic mean is calculated as the square root of the mean of the of the given numbers, then the average $Q$ is defined by the formula:

$$
Q=\sqrt{\frac{x_{1}^{2}+x_{2}^{2}+\cdots+x_{2}^{2}}{n}}
$$

Let $\mathbf{F}$ is a finite field with q elements. 
The Hamming distance[8] d $(\underline{x}, \underline{y})$ between two vectors $\underline{x}, \underline{y} \in$ $\mathrm{F}^{(\mathrm{n})}$ is the number of coefficients in which they differ,

$d(x, y)=\operatorname{Card}\left\{i / x_{i} \neq y_{i}\right\}$

The Degree of difference [8]: Given the real values $x$ and $y$, the degree of difference between $x$ and $y$, modulated by $\alpha>0$, denoted by $\alpha_{\alpha}(x, y)$ is defined as:

$d_{E}(x, y)=1-e^{-\alpha[x-y]^{2}}$

The parameter a modulates the degree of difference in the sense that for the same value of $|x-y|$, different values of will result in different values of $d_{g}(x, y)$

\section{The Difference fuzzy set for two vectors[8]}

Let $x$ and $y$ be twon-dimensional real vectors, and let $x i, y i$ denote their corresponding ith component. The degree of difference between $x$ and $y$ along the component $i$, modulated by the parameter $a$ is $d_{\alpha}\left(x i, y_{i}\right)$. The difference fuzzy set corresponding to $d_{\alpha}\left(x w_{i}, y\right)$ is $D_{\alpha}(x, y)$ with membership function:

$H_{D_{x}}(x, y)(i):\{1, \ldots, n\} \rightarrow[0,1]$

$\mu D_{\alpha}(x, y)(i)=d_{g}(x i, y i)$

$\mu D_{\alpha}(x, y)(i)$ is the degree to which the vectors $x$ and $y$ are different along there ith component.

\section{The Cardinality of a fuzzy set [8]:}

Let: $\quad A \equiv \sum_{i=1}^{n} x i / \mu i$

denote the discrete fuzzy set $A$ over the universe of discourse $[x 1, \ldots, x n]$ where $\mu i=\mu A(x i)$ denotes the degree of membership for $x$ to $A$. The cardinality, $\operatorname{Card} A$, of $A$ is a

fuzzy set: $\quad \operatorname{Car} d A \equiv \sum_{i=1}^{n} \frac{i}{\mu_{\operatorname{Card}(A)}(i)}$

Where: $\quad \mu_{\text {Card }}(A)=\mu_{\{0} n\left(1-\mu_{(i+1)}\right)$

Where $\mu_{(i)}$ denotes the $i$ th largest value of $\mu \mathrm{i}$, the values

$\mu_{(0)}=1$ and $\mu_{(n+1)}=0$ are introduced for

convenience, and ${ }^{\wedge}$ denotes the min operation.

the non-fuzzy cardinality $n C \operatorname{ard}(A)$,is:

$n \operatorname{Cand}(A)=\operatorname{cand} \overline{\left(x, \mu_{\mathrm{C}}(x)>0.5\right)}$

where for a set $5, \bar{S}$ denotes the closure of $S$.

The fuzzy Hamming distance [8]between $x$ and $y$, denoted by $F H D_{s}(x, y)$ is the fuzzy cardinality of the difference fuzzy set, $D_{\alpha}(x, y)$ :

$\mu_{\text {FHD }}\left(x_{n} n(\alpha)=\{1, \ldots, n] \rightarrow[0,1]\right.$

$\mu_{F H D(x, n}(k, \alpha)=\mu_{\operatorname{CordD}_{\alpha}\left(x_{n}\right)}(R)$

For $k \in\{1, \ldots, n)$ where $n=\| 5$ upport $D_{\alpha}(x, y) \|$ for a given value $k, \mu_{F H D\left(x_{y}\right)}(k, \alpha)$ is the degree to which the vectors $x$ and $y$ are different on exactly $k$ components (with the modulation constant $\alpha$ ).

Based on the rules used to choose the perfect face, based on the ideas of the famous Italian doctor one of the fonder of criminology and based also on THE IDEAL PROPORTIONS OF THE FACE described by rhino plastic doctors, the ratios of the distances taken as the scientific measuring guns of the beauty in a perfect face are calculated. the same distances are computed for each face sketch in dataset of the face sketches. figure 1 illustrated the Scientifics rules used to select the beauty queen.

The different distances used to calculate the proportions of the face are inspired by cannons in a perfect face. We define the following distances as:

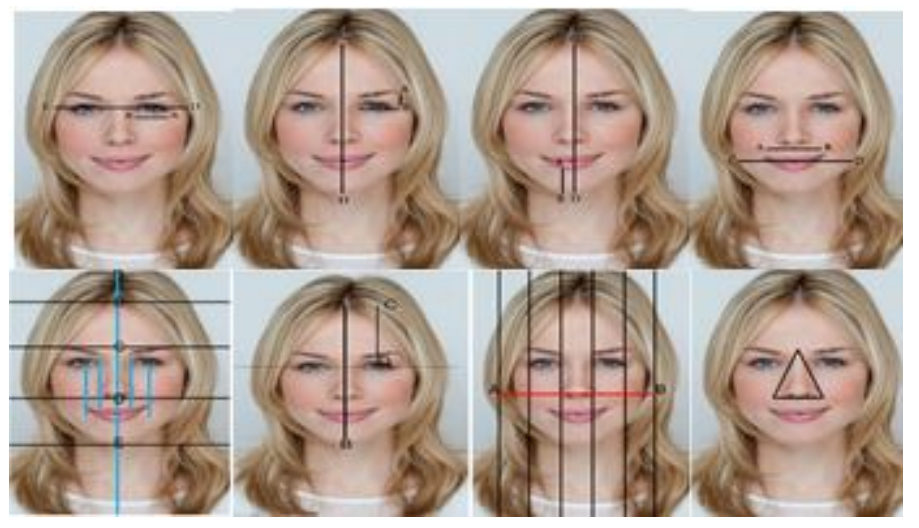

Figure1. Photos of Colgate, an 18-year-old Briton, won a beauty pageant where the contestants were judged on scientific criteria. The scientific rules used from left to right and from top to bottom: $A B=1 / 5 C D, A B=1 / 10 C D$, $\mathrm{AB}=1 / 5 \mathrm{CD}, \mathrm{AB}=1 / 2 \mathrm{CD}, \mathrm{BC}=\mathrm{CD}=\mathrm{DE}=1 / 3 \mathrm{BE} ; \mathrm{AB}=1 / 2 \mathrm{BC}, \mathrm{CD}=1 / 2$ $\mathrm{AB}, \mathrm{AB}=5$ eyes, nose surface $<=5 \%$ total of facial surface.

d0: The face width

d1: The distance between the end of the right eye and the right end of the face

d2: The length of the right eye

d3: The distance between the eyes

d4: The length of the left eye

d5: The distance between the end of the left eye and the left end of the face

d6: The distance between the centers of the pupils

d7: The mouth length

d8: The distance between eyes

d9: The nose width

d10: The mouth length

d11: The jaw length

d12: The distance between the eyes and the last point of the head

d13: The distance between the eyes and the chin

d14: distance between the center of the forehead and the last point of the head

d15: The distance between the center of the forehead and the nose

d16: The distance between nose and the chin 
d17: The distance between the eye and the eyebrow

d18: The length of one eye

d19: The distance between the low lip and the chin

d20: The facial length

\section{APPROACH}

Our system has two modes, in both them, the input facial emotion photo and all faces emotion photos of the dataset are converted to a Gray level, they are resized and cropped into $200 \times 250$ pixels. These dimensions are chosen: It's the proposed default choice of the datasets used and it's also the dimensions used in related works. The Haar-cascades [12][14] are used for detecting the face in each photo image. The first step of the system is to pretrain and to normalize all the emotional photos in the offline phase. For that they have been transformed into a gray level image and are all cropped to $200 \times 250$ pixels. The same technique is thus used to online mode. After this step, we projected the famous algorithm of viola and jones to detect the faces of the images. The process that follows this second step is used to locate the 68_point_landmarks in each face. These 68 points [13][14] will be the parameter of our descriptor which allows to extract an identity of each face via the calculation of the rations of the perfect face. A vector will be dedicated to group these harmonious distances in order. This vector represents a real proportionality with any other similar vector. The ratios of these distances in the vector have been stored as already detailed in this section.

An overview of our proposed Fuzzy Hamming Distance based framework for face sketch recognition is shown in Figure 2.

In online process of the Face Emotion Recognition System, given a facial emotion, sixteen features are extracted. The series of these characteristics composes a vector of real values. This vector is considered as an identifier of the face from which the values have been extracted and calculated.

In offline process of the Facial Emotion Recognition System, we grouped the dataset of facial emotion photos into seven data subsets based on the kind of emotion that it represents. The same distances (used in online process) are extracted and calculated for each facial emotion photo for each data subset in the facial emotion dataset. We generate seven referential faces suitable for each kind of facial emotion based on the classical means or averages: Arthritic mean, Contra harmonic mean, Geometric mean, Harmonic mean and quadratic mean. We use the landmarks points the generate all referential faces.

After such a facial emotion photos and referential faces transformation to vectors of reals values, Facial emotion recognition becomes straightforward. We can compare the facial emotion with the referential facial emotions using the Fuzzy Hamming Distance. In fact, we first compute the landmarks points for each facial emotion image and also for all referential faces. The face ratios for each one is then used as feature vectors for final classification and recognition.

The detail algorithm can be summarized as follows.

(1): To pretrain and to normalize all the facial emotion photos

(2): Extract the landmarks points of the facial emotion photos according to the model "The 68_face_landmarks"

(3): Calculate the distances $\mathrm{d}_{0}$ to $\mathrm{d}_{20}$ as defined in section 4 . we assume that:

$$
\begin{array}{llll}
x_{1}=\frac{d 1}{d 0} & x_{2}=\frac{d 2}{d 0} & x_{3}=\frac{d 3}{d 0} & x_{4}=\frac{d 4}{d 0} \\
x_{5}=\frac{d 5}{d 0} & x_{6}=\frac{d 6}{d 7} & x_{7}=\frac{d 8}{d 9}, & x_{8}=\frac{d 10}{d 11} \\
x_{9}=\frac{d 12}{d 13} & x_{10}=\frac{d 14}{d 20} & x_{11}=\frac{d 15}{d 20} & x_{12}=\frac{d 16}{d 20} \\
x_{13}=\frac{d 17}{d 18} & x_{14}=\frac{d 19}{d 20} &
\end{array}
$$

(4): Calculate $S$ : the surface of the nose (See Figure 2)

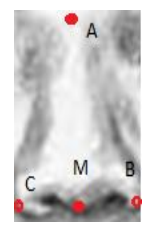

Figure2. The principle used for the calculation of the surface of the nose $S$ is based on the Manhattan distance used here to calculate the sides of the triangle encompassing the nose

The Manhattan distance is used here to calculate the sides of the triangle encompassing the nose.

$s=\frac{1}{4} \sqrt{(a+b+c)(a+b-c)(-a+b+c)(a-b+c)}$

We define: $\quad x_{15}=s$

(5) let:

$\mathrm{E}=$ The tragus center of the ear

$\mathrm{I}=$ Center inter eyebrow

$\mathrm{F}=$ Center of the front

$\mathrm{C}=$ The chin

$\mathrm{N}=$ Dorsum of nose

The following angles are calculated:

$$
\begin{aligned}
\theta 1 & =\overline{F E I}=\frac{\arccos \left(E F^{2}+E I^{2}-F I^{2}\right)}{2 * E F * E I} \\
\theta 2 & =\overline{I E N}=\frac{\arccos \left(E N^{2}+E I^{2}-N I^{2}\right)}{2 * E N * E I} \\
\theta 3 & =\overline{N E C}=\frac{\arccos \left(E N^{2}+E C^{2}-N C^{2}\right)}{2 * E N * E C} \\
x_{16} & =\frac{\theta 1+\theta 2}{\theta 3}
\end{aligned}
$$


Then:

$$
x_{36}=\frac{\frac{a r c \cos \left(E F^{2}+E I^{2}-F I^{2}\right)}{2 * E F * E I}+\frac{a r c \cos \left(E N^{2}+E I^{2}-N I^{2}\right)}{2 * E N * E I}}{\frac{a r c \cos \left(E N^{2}+E C^{2}-N C^{2}\right)}{2 * E N * E C}}
$$

(6) Create the vector:

$$
V=x i, h \in\{1, \ldots, 16\}
$$

(7): Compute FHD between Vi (the vector of the input facial emotion photo) and each vector $\mathrm{Vf}$ in the list of referential faces vectors; Their FHD is the same as that between the vector 0 and $|x-y|$. Therefore, the FHD between $x$ and $y$ is the cardinality of the fuzzy Return setD $(|x-y|, 0)$.

(8): Recognition: The output is the kind of emotion of the min distance for the referential face parameter.

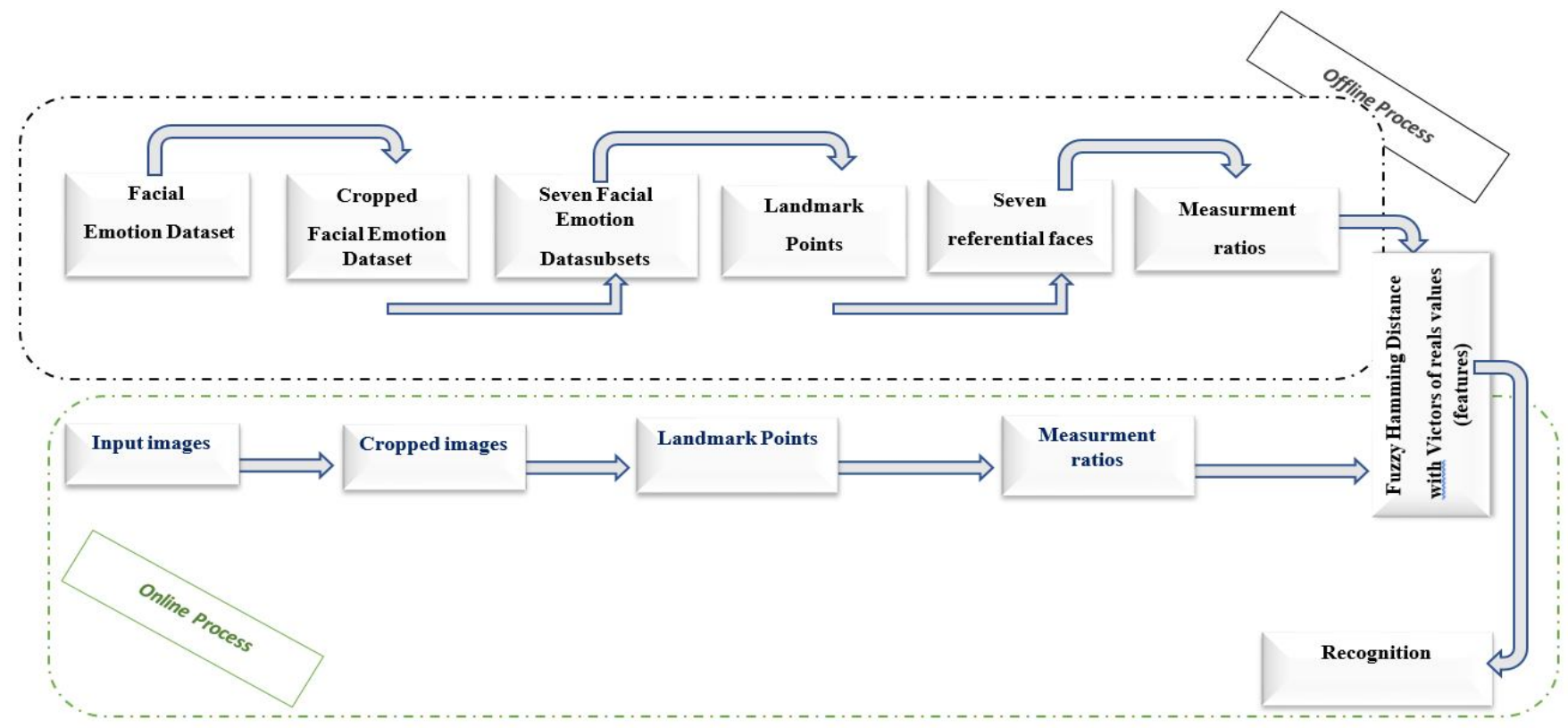

Figure3. An overview of our proposed framework baseed on fuzzy hamming distance with face ratios for facial emotion recognition: Given an input facial emotion: Converted to gray level and cropped to 200x250 the facial emotion(200x250 is the standard size chosen in The relative database), detected the face and localized 68 landmarks points. Sixteen features are calculated based on the portions of the perfect face and an array of them is generated. These steps are similar between the two modes of facial emotion recognition system FER. In offline process of FER , the system can associate the right kind emotion for each input image: The parameters of Fazzy Hamming distance are the extracted features from the facial emotion image and the referential faces, on the other side of the system , the generated vector from the facial emotion is involved to recognize its kind of emotion. The output result of our FER is the probe kind of emotion.

\section{EXPERIMENTS AND RESULTS}

To demonstrate the $\mathrm{e} \square$ ectiveness of the proposed method, we proceeded for acquiring the dataset of emotional facial expression pictures. We used WSEFEP [15][16] dataset, the Warsaw Set of Emotional Facial Expression Pictures. The WSEFEP is comprised of those pictures that received the highest recognition marks (e.g., accuracy with intended display) from independent judges, totaling 210 high quality photographs of 30 individuals.

We divide the dataset to seven data subsets suitable for each kind of basics kinds of emotions: Anger, Disgust, Fear, Happiness, Neutral, Sadness and Surprise, TABLE 1 shown the percentage of each kind of emotions.

Table 1: The percentage of each kind of emotions from WSEFEP dataset
The WSEFEP is used to training and to testing our approach. Figure 5 clearly illustrates step by step the results obtained as

\begin{tabular}{|c|c|c|c|c|c|c|c|}
\hline 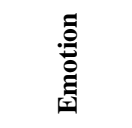 & $\begin{array}{l}\bar{D} \\
\dot{0} \\
\bar{z}\end{array}$ & 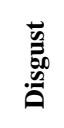 & 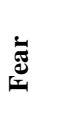 & 怘 & 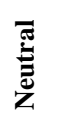 & $\frac{\mathscr{E}}{\tilde{E}}$ & 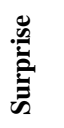 \\
\hline Percentage & $\begin{array}{c}14.28 \\
\%\end{array}$ & $\begin{array}{c}14.28 \\
\%\end{array}$ & $\begin{array}{c}14.28 \\
\%\end{array}$ & $\begin{array}{c}14.28 \\
\%\end{array}$ & $\begin{array}{c}14.28 \\
\%\end{array}$ & $\begin{array}{c}14.28 \\
\%\end{array}$ & $\begin{array}{c}14.28 \\
\%\end{array}$ \\
\hline
\end{tabular}
we progress in the process of the framework already described

previously. In the first step the extract emotional photos of the dataset. In the second: The cropped photos. In the third: The extract 68 face landmarks points. In the fourth:

calculate of the sixteen features and in the last step: the vector generated from the facial emotion photos. It is involved to recognized the kind of the facial emotion in the input photo. The output result of FER is the probe kind of emotion. 
We generate seven referential faces suitable for each kind of facial emotion based on perfect face ratios and five classical averages: arithmetic mean, geometric mean, harmonic mean, contraharmonic mean and quadratic mean. The result is 35 referential faces. Figure 4 shown our referential face landmarks photo for each type of average. The images for the usual means are in bleu and they are in red for the author means.

Using our new method, we realized five experiments according to the average used during the generation of the referential face. In each one of them, we compare the input facial emotion image (features) with the seven referential faces, for that we used the Fuzzy Hamming distance. The output probe kind of emotion is that proper to the referential face having the minimal distance. TABLE 1 shows the cumulative match scores for our approach based on Fuzzy Hamming Distance (FHD) and Average Face Ratios. The result can be considered as a benchmark for the facial emotion recognition system to compare. All experimental results of tests are shown in Figure 6. 
Khalid Ounachad et al., International Journal of Advanced Trends in Computer Science and Engineering, 9(3), May - June 2020, 3417 - 3425

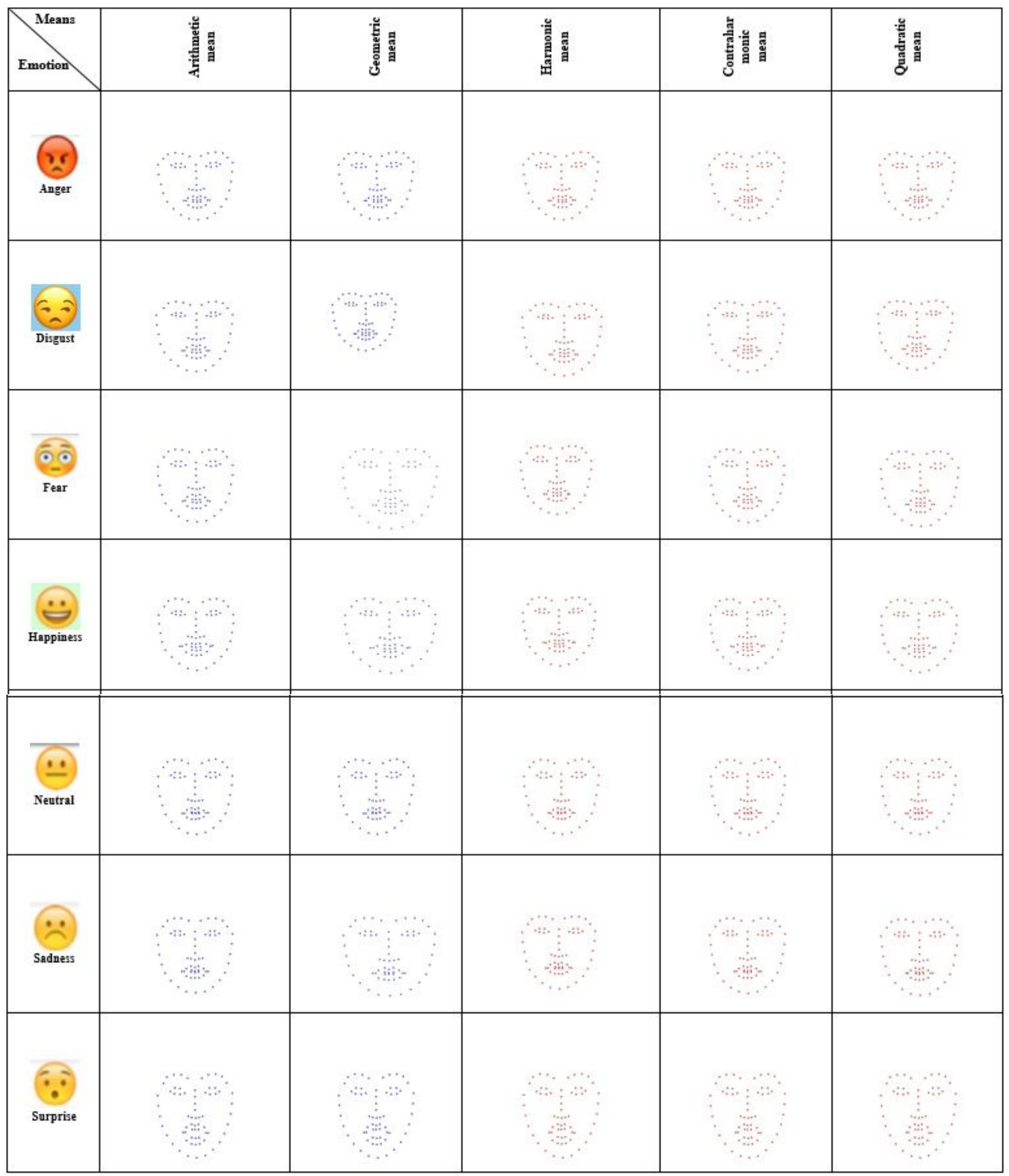

Figure4. Our referentiels 68_face emotion_landmarks attributed to their kind of emotion and the kind of mean used to calculate its., each model containbed: "Mouth", "Right_Eyebrow", "Left_Eyebrow", "Right_Eye","Left_Eye", "Nose" and "Jaw".The images for the usuel means are in bleu and they are in red for the auther means. 


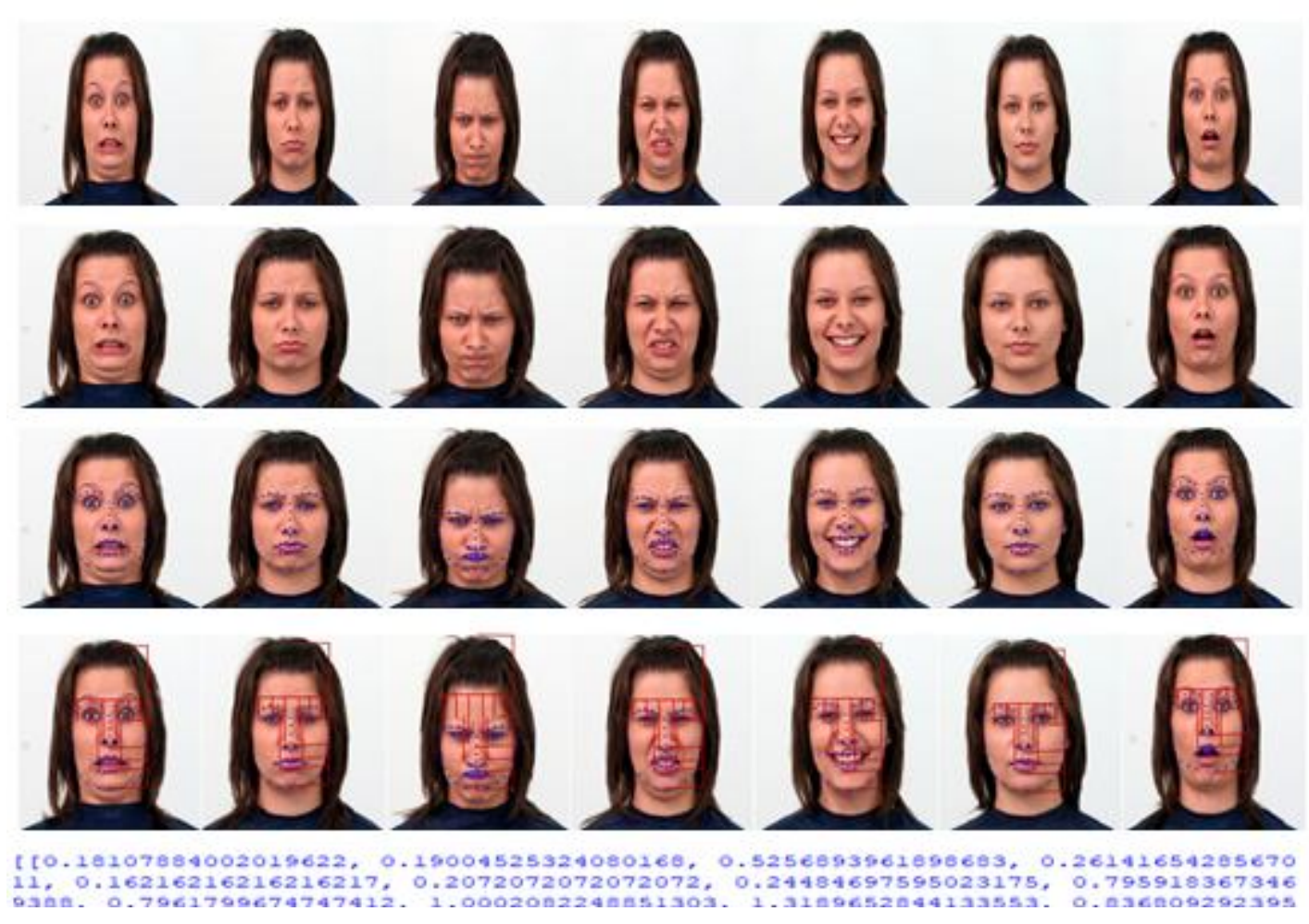

Figure5. The process of our FSR, line1: extract of the dataset photos/input facial emotion. Line2: cropped emotional photos. line3: extract the 68 face landmarks points. Line 4: calculate of sixteen features and line5: the vector generated from the facial emotion photos, it is involved to recognized the kind of emotion. The output result of FER is the probe kind of emotion of the input facial photos

All experimental results of tests are shown in Figure 6.

The cumulative match score is used to evaluate the performance of the algorithms. It measures the percentage of the probe emotion.

Table 2: The cumulative match scores for our approach based on

\begin{tabular}{|c|c|c|c|c|c|c|c|}
\hline Emotion & 总 & 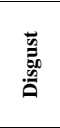 & છี & 离 & 焉 & 㲵 & 总 \\
\hline $\begin{array}{c}\text { Arithmetic } \\
\text { Mean }\end{array}$ & 50.00 & 36.67 & 43.34 & 86.67 & $\underline{73.34}$ & $\underline{50.00}$ & $\underline{86.67}$ \\
\hline $\begin{array}{c}\text { Geometric } \\
\text { Mean }\end{array}$ & 56.67 & $\underline{46.67}$ & $\underline{46.67}$ & 90.00 & $\underline{73.34}$ & 33.34 & $\underline{86.67}$ \\
\hline $\begin{array}{l}\text { Harmonic } \\
\text { Mean }\end{array}$ & 60.00 & 40.00 & 36.67 & $\underline{93.34}$ & $\underline{73.34}$ & 36.67 & $\underline{86.67}$ \\
\hline $\begin{array}{c}\text { Contra } \\
\text { Harmonic } \\
\text { mean }\end{array}$ & $\underline{63.34}$ & 36.67 & 40.00 & $\underline{93.34}$ & $\underline{73.34}$ & 36.67 & $\underline{86.67}$ \\
\hline $\begin{array}{l}\text { Quadratic } \\
\text { Mean }\end{array}$ & 60.00 & 36.67 & 40.00 & 93.34 & $\underline{73.34}$ & 33.34 & $\underline{86.67}$ \\
\hline
\end{tabular}

Fuzzy Hamming Distance (FHD) and Average Face Ratios (AFR)

TABLE 2 reports the facial emotion recognition accuracies using five different methods: arithmetic mean, geometric mean, harmonic mean, contra harmonic mean and quadratic mean. Our algorithm proves that, the recognition rate reaches more than $63 \%$ for angry emotion, more than $46 \%$ for disgust and fear emotions, more than $93 \%$ for happiness emotion, more than $73 \%$ for neutral emotion, $50 \%$ for sadness emotion and more than 86.67 .

The figure. 6 shows the cumulative match scores for our approach. The $\mathrm{x}$-axis represents the kind of emotion and the $y$-axis represents the recognition rate. The results clearly demonstrate the superiority of our algorithm to recognize the happiness emotion but the last rate of recognition is that of sadness emotion. The quadratic mean helps to better recognize the happiness emotion but not able to better recognize the disgust and sadness emotions. The contraharmonic mean helps to better recognize the angry emotion. The geometric mean helps to better recognize disgust and fear emotions. The arithmetic mean helps to better recognize the sadness emotion and neutral and surprise emotions are recognized with the same rate for the all averages.

We tested our approach with five different facial emotion recognition methods using classical averages and Fuzzy Hamming distance. In these methods, the cumulative match score proves the performance of the algorithms. The 
recognition rate reaches more than $93 \%$ in WSEFEP dataset. The algorithmic complexity of our approach is $\mathrm{O}(\mathrm{n})$.

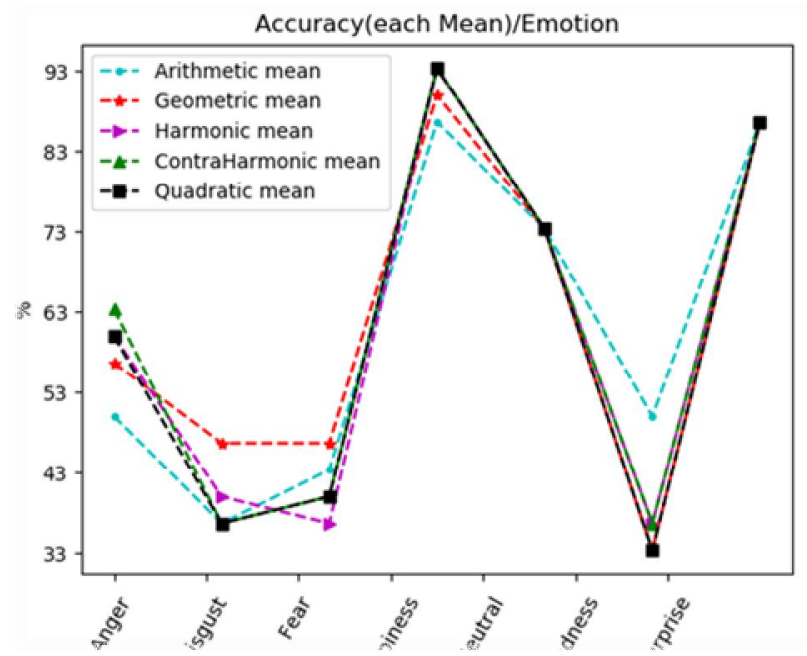

Figure6. Comparison of cumulative match scores between our various facial emotion recognition methods using five classical averages

\section{CONCLUSION}

This paper proposes a new geometrical method for facial emotion recognition. The methods are based on Fuzzy Hamming Distance and the Referential Face Ratios. We used sixteen features based on the distance between different portions of the perfect face. We tested our method on WSEFEP dataset and the results is very satisfactory. Our work is inspired by the recent successful methods that showed that relatively simple features could be used to give good performance in a Fuzzy Hamming Distance -based framework. The future work will include a decrease in the number of the features used.

\section{REFERENCES}

1. P. Ekman and W. V. Friesen. Universals and cultural differences in the judgements of facial expressions of emotion, Journal of Personality and Social Psychology, Vol. 53, pp. 712-717, November. 1987. https://doi.org/10.1037/0022-3514.53.4.712

2. K. Feng and T. Chaspari. A Review of Generalizable Transfer Learning in Automatic Emotion Recognition, Front. Comput. Sci. Vol. 28. February 2020 https://doi.org/10.3389/fcomp.2020.00009

3. S. asif Hussain, A. Balushi. A real time face emotion classification and recognition using deep learning model, Journal of Physics: Conference Series, Journal of Physics: Conference Serie. Vol.1432. January 2020

4. C. Sukhada, MS. Sannidhan, K. B Sudeepa and B.Abhir. A Study on various state of the art of the Art Face Recognition System using Deep Learning Techniques, International Journal of Advanced Trends in Computer
Science and Engineering, Vol.8, pp.1590-1600. July-August 2019.

https://doi.org/10.30534/ijatcse/2019/84842019

5. H. Almohamedh and S. Almotairi. Facial Emotion Recognition Using Eigenface and Feature Optimization, International Journal of Advanced Trends in Computer Science and Engineering, Vol.8, pp.1181-1185. July-August 2019. https://doi.org/10.30534/ijatcse/2019/28842019

6. A.Bookstein,S.T.Klein and T.Raita. Fuzzy Hamming Distance: A New Dissimilarity Measure, Lecture Notes in Computer Sciences, 2089, pp. 86-97. 2006

7. K.Ounachad, A.Sadiq and A.Souhar. Fuzzy Hamming distance and Perfect Face Ratios based Face Sketch Recognition, IEEE CIST'18, pp.317-322, Oct 2018. https://doi.org/10.1109/CIST.2018.8596665

8. M.Ionescu and A.Ralescu. Fuzzy Hamming Distance Based Banknote Validator, IEEE International Conference on Fuzzy Systems,pp. 300-305, June 2005.

9. M.Ionescu and A. Ralescu. Image clustering for a fuzzy hamming distance based cbir system. In Proceedings of the Sixteen Midwest Artificial Intelligence and Cognitive Science Conference. Dayton, pp 102-108, April 2005.

10. M.Ionescu and A.Ralescu. Fuzzy Hamming Distance in a Content-Based Image Retrieval System, IEEE International Conference on Fuzzy Systems, Vol.3, pp.1721-1726, August 2004.

11. A.M.Ashir, G.S.Shehu and A.Eleyan. Character Recognition Using Correlation \& Hamming Distance, Signal Processing and Communications Applications Conference (SIU), 23th edition, pp. 755-758. May 2015.

12. M. Oualla, A. Sadiq and S. Mbarki. Comparative study of the methods using Haar-Like features. International journal of engineering sciences \& research. Vol.4, pp.35-43. April 2015

13. P. Viola and M.J. Jones. Robust Real-Time Face Detection. International Journal of Computer Vision. Vol.57, pp.137-154. May 2004

14. C. Rahmad, R. A. Asmara, D. R. H. Putra, I. Dharma, H. Darmono and I. Muhiqqin. Comparison of Viola-Jones Haar Cascade Classifier and Histogram of Oriented Gradients (HOG) for face detection, IOP Conf. Series: Materials Science and Engineering.Vol. 732, January 2020

15. M. Olszanowski, G. Pochwatko, K. Kuklinski, M. S.Rylski, P. Lewinski and R. K. Ohme. Warsaw set of emotional facial expression pictures: a validation study of facial display photographs, Frentiers in psychology Emotion science, 5:1516. January 2015 https://doi.org/10.3389/fpsyg.2014.01516

16. L. Kulke, D. Feyerabend and A.Schacht. A Comparison of the Affectiva iMotions Facial Expression Analysis Software with EMG for Identifying Facial Expressions of Emotion, Frontiers in Psychology. Vol.11. February 2020 